\title{
A Comparative Study of Nanowire Arrays for Maximum Power Transmission
}

\author{
Hasan Aykut Şatana, Barışcan Karaosmanoğlu and \\ Özgür Ergül
}

Additional information is available at the end of the chapter

http://dx.doi.org/10.5772/67447

\begin{abstract}
In this chapter, we present a comparative study of nanowire arrays for the purpose of transmitting electromagnetic energy to long distances with minimum loss. Silver nanowires at an infrared frequency are considered as a case study, using an accurate simulation environment based on the surface-integral equations and the multilevel fast multipole algorithm (MLFMA). Reliable numerical results are obtained by considering arrays as three-dimensional structures with finite sizes in all dimensions. Nanowires with different cross sections are compared in alternative array configurations to assess and compare their transmission properties. While there are no significantly varying performances for the arrays of few elements, large discrepancies occur as the number of nanowires increases. We show that arrays involving nanowires with hexagonal cross sections in hexagonal arrangements can provide significantly better power transmissions in comparison to others. We also present the superiority of a particular case, where the nanowires and gaps between them, both with hexagonal cross sections, are equally distributed, leading to a honeycomb structure. This type of structures demonstrates high-quality transmissions that can be useful in diverse application areas, such as optical coupling, subwavelength imaging, and energy harvesting.
\end{abstract}

Keywords: nanowire arrays, optical power transmission, surface integral equations, multilevel fast multiple algorithm

\section{Introduction}

Nanowires are excellent tools for guiding electromagnetic waves with minimum loss at optical frequencies [1-4] particularly as an alternative to dielectric structures at nanometer scales. Plasmonic properties of metals, such as silver $(\mathrm{Ag})$ and gold $(\mathrm{Au})$, enable high-quality power transmissions along nanowires via surface plasmon polaritons [5]. In addition to their favorable characteristics that fully exploit the benefits of the surface plasmons to transfer 
electromagnetic energy to long distances [6, 7], nanowires are relatively easier to fabricate, making them popular in diverse optics applications, such as optical coupling [4], subwavelength imaging [8-10], microscopy and sensing [11-13], and energy harvesting [14, 15]. While the first samples of fabricated nanowires in the literature are often deformed with undesired defects on their surfaces, rapid advances in nanotechnology have enabled the fabrication of progressively improved structures with desired geometric features. Recent studies demonstrate not only isolated nanowires with smooth surfaces but also their periodic arrangements in regular and highly ordered forms $[16,17]$.

As in all subareas of nanotechnology, experimental studies on nanowires are supported by numerical and computational analysis $[18,19]$. The plasmonic properties of metals at optical frequencies can be modeled using tabulated or formulated permittivity values [20] inserted into the conventional solvers for penetrable bodies [21]. Accurate simulation environments based on various methods and techniques can provide an ability to investigate the effects of the cross-sectional shapes [22-24] and the choice of the material [22] on the nanowire characteristics before their fabrications. For plasmonic problems, not only limited to nanowires, computational methods based on the differential equations are very popular in the literature due to their availability as commercial and noncommercial software. On the other hand, for nanowire simulations, these methods may not enable a rigorous analysis, as the nanowire arrays are typically long in terms of wavelength, while they must be modeled as finite to accurately quantify their electromagnetic responses in most cases. In addition, in array configurations, several nanowires must be modeled together by considering all interactions and coupling between them, without employing any periodicity and/or infinity. For this purpose, integral-equation formulations, particularly the surface-integral equations [25-31] that need discretizations only on the surfaces of the structures, are very suitable for the computational analysis of nanowires, as well as similar structures.

In parallel to the improved capabilities in nanotechnology, recent studies focus on geometric properties (e.g., cross section and length) of nanowires to maximize their performances in the related applications [32,33]. On the other hand, the best geometric properties of the nanowires, especially their cross sections, and their arrangements in array configurations for maximizing the power output in transmission lines are not well known. In this chapter, we present a comparative study of nanowire arrays in terms of power transmission, that is, transfer of the electromagnetic energy with minimum loss. For numerical tests, we particularly consider transmission properties of Ag nanowires at an infrared frequency $(250 \mathrm{THz})$ and compare various cross-sectional geometries in alternative arrangements. An accurate solver based on surface-integral equations and the multilevel fast multipole algorithm (MLFMA) [34, 35] is used to analyze nanowires as three-dimensional finite structures with full parametric modeling of metals using measured values of the electrical permittivity. Surface formulations lead to dense matrix solutions, whose solutions can be challenging due to the ill-conditioned nature of the problems, large negative permittivity values at optical frequencies, and large dimensions in terms of the operation wavelength. These challenges are solved by using MLFMA in an inner/ outer iterative scheme. With the high efficiency and accuracy of the developed solver, we analyze realistic structures by including all mutual couplings between the interacting nanowires. The results demonstrate interesting properties of hexagonal arrangements of 
hexagonal cells, leading to honeycomb structures, which demonstrate much better transmission capabilities in comparison to others when the arrays are relatively large. In the literature, it is shown that such hexagonal arrangements of nanowires can indeed be fabricated [13, 17], while we also show that the periodicity and the size of the nanowires (that may bring additional challenges in the fabrication) are also critical on the transmission performances of the arrays.

This chapter is organized as follows. First, in Section 2, we briefly present the simulation environment used in this study with some details of the major components. Parameters and variables of the nanowire simulations are discussed in Section 3. Then, Section 4 includes numerical results and comparisons, followed by the discussion and concluding remarks in Sections 5 and 6 .

\section{Simulation environment}

The developed simulation environment for the analysis of plasmonic problems, such as nanowires at optical frequencies, is extensively discussed in Ref. [36]. In this section, we consider the major components of the solver for accurate and efficient analysis of nanowires and their arrays.

\subsection{Modified combined tangential formulation}

Consider a homogeneous object (nanowire or nanowire array) located in free space (vacuum) and illuminated by time-harmonic sources described by incident electric and magnetic fields $\left(\boldsymbol{E}^{\mathrm{inc}}\right.$ and $\left.\boldsymbol{H}^{\mathrm{inc}}\right)$. The object is assumed to be nonmagnetic with permeability $\mu_{o}=4 \pi \times 10^{-7} \mathrm{H} / \mathrm{m}$ and characterized by a permittivity $\varepsilon_{p}=\left(-\varepsilon_{R}+i \varepsilon_{I}\right) \varepsilon_{0}$, where $\varepsilon_{o}=1 /\left(c^{2} \mu_{0}\right)$ and $c=299792458 \mathrm{~m} / \mathrm{s}$. The equivalence theorem allows us to formulate the problem with equivalent currents $\boldsymbol{J}=\hat{n} \times \boldsymbol{H}$ and $\boldsymbol{M}=-\hat{n} \times \boldsymbol{E}$ defined on the surface of the object with outward normal $\hat{n}$. In phasor domain with the $\exp (-i \omega t)$ time convention, these currents radiate in homogeneous spaces using the impulse (Green's) functions $g_{o, p}\left(\boldsymbol{r}, \boldsymbol{r}^{\prime}\right)=\exp \left(i k_{o, p}\left|\boldsymbol{r}-\boldsymbol{r}^{\prime}\right|\right) /\left(4 \pi\left|\boldsymbol{r}-\boldsymbol{r}^{\prime}\right|\right)$, where $k_{o}=\omega \sqrt{\mu_{o}} \sqrt{\varepsilon_{o}}$ and $k_{p}=\omega \sqrt{\mu_{o}} \sqrt{\varepsilon_{p}}$ are the wavenumbers for the angular frequency $\omega=2 \pi f=2 \pi c / \lambda_{o}$ (hence, $\lambda_{o}$ is the wavelength in free space). Outer and inner problems are superimposed using the boundary conditions, that is, continuity of the tangential electric and magnetic field intensities, leading to a set of equations that are discretized and converted into matrix equations for numerical solutions.

The method to enforce the boundary conditions leads to alternative ways to construct formulations. Among many, we prefer a recently developed modified combined tangential formulation (MCTF), which can be written as

$$
\left[\begin{array}{ll}
\overline{\mathbf{Z}}_{11}^{\mathrm{MCTF}} & \overline{\mathbf{Z}}_{12}^{\mathrm{MCTF}} \\
\overline{\mathbf{Z}}_{21}^{\mathrm{MCTF}} & \overline{\boldsymbol{Z}}_{22}^{\mathrm{MCTF}}
\end{array}\right] \cdot\left[\begin{array}{c}
\boldsymbol{a}_{J} \\
\boldsymbol{a}_{M}
\end{array}\right]=\left[\begin{array}{l}
\boldsymbol{w}_{1}^{\mathrm{MCTF}} \\
\boldsymbol{w}_{2}^{\mathrm{MCTF}}
\end{array}\right],
$$


where

$$
\begin{aligned}
& \bar{Z}_{11}^{\mathrm{MCTF}}[m, n]= i \omega \mu_{o} \int_{S_{m}} d \boldsymbol{r} \boldsymbol{t}_{m}(\boldsymbol{r}) \cdot \int_{S_{n}} d \boldsymbol{r}^{\prime} \boldsymbol{b}_{n}\left(\boldsymbol{r}^{\prime}\right)\left[g_{o}\left(\boldsymbol{r}, \boldsymbol{r}^{\prime}\right)+g_{p}\left(\boldsymbol{r}, \boldsymbol{r}^{\prime}\right)\right] \\
&+\frac{i}{\omega} \int_{S_{m}} d \boldsymbol{r} \boldsymbol{t}_{m}(\boldsymbol{r}) \cdot \int_{S_{n}} d \boldsymbol{r}^{\prime} \nabla^{\prime} \cdot \boldsymbol{b}_{n}\left(\boldsymbol{r}^{\prime}\right)\left[\frac{1}{\varepsilon_{0}} \nabla g_{o}\left(\boldsymbol{r}, \boldsymbol{r}^{\prime}\right)+\frac{1}{\varepsilon_{p}} \nabla g_{p}\left(\boldsymbol{r}, \boldsymbol{r}^{\prime}\right)\right] \\
& \overline{\mathbf{Z}}_{12}^{\mathrm{MCTF}}[m, n]=- \int_{S_{m}} d \boldsymbol{r} \boldsymbol{t}_{m}(\boldsymbol{r}) \cdot \int_{P V, S_{n}} d \boldsymbol{r}^{\prime} \boldsymbol{b}_{n}\left(\boldsymbol{r}^{\prime}\right) \times\left[\nabla^{\prime} g_{o}\left(\boldsymbol{r}, \boldsymbol{r}^{\prime}\right)+\nabla^{\prime} g_{p}\left(\boldsymbol{r}, \boldsymbol{r}^{\prime}\right)\right] \\
& \bar{Z}_{21}^{\mathrm{MCTF}}[m, n]=-\frac{\mu_{o}}{\sqrt{\varepsilon_{o}} \sqrt{\varepsilon_{p}}} \overline{\mathbf{Z}}_{12}^{\mathrm{MCTF}}[m, n] \\
& \overline{\boldsymbol{Z}}_{22}^{\mathrm{MCTF}}[m, n]=i \omega \mu_{o} \int_{S_{m}} d \boldsymbol{r} \boldsymbol{r}_{m}(\boldsymbol{r}) \cdot \int_{S_{n}} d \boldsymbol{r}^{\prime} \boldsymbol{b}_{n}\left(\boldsymbol{r}^{\prime}\right)\left[\frac{\sqrt{\varepsilon_{0}}}{\sqrt{\varepsilon_{p}}} g_{o}\left(\boldsymbol{r}, \boldsymbol{r}^{\prime}\right)+\frac{\sqrt{\varepsilon_{p}}}{\sqrt{\varepsilon_{o}}} g_{p}\left(\boldsymbol{r}, \boldsymbol{r}^{\prime}\right)\right] \\
&+\frac{i}{\omega \sqrt{\varepsilon_{o}} \sqrt{\varepsilon_{p}}} \int_{S_{m}} d \boldsymbol{r} \boldsymbol{r}_{m}(\boldsymbol{r}) \cdot \int_{S_{n}} d \boldsymbol{r}^{\prime} \nabla^{\prime} \cdot \boldsymbol{b}_{n}\left(\boldsymbol{r}^{\prime}\right)\left[\nabla g_{o}\left(\boldsymbol{r}, \boldsymbol{r}^{\prime}\right)+\nabla g_{p}\left(\boldsymbol{r}, \boldsymbol{r}^{\prime}\right)\right]
\end{aligned}
$$

and

$$
\begin{gathered}
\boldsymbol{w}_{1}^{\mathrm{MCTF}}[m]=-\int_{S_{m}} d \boldsymbol{r} \boldsymbol{t}_{m}(\boldsymbol{r}) \cdot \boldsymbol{E}^{\mathrm{inc}}(\boldsymbol{r}) \\
\boldsymbol{w}_{2}^{\mathrm{MCTF}}[m]=-\frac{\mu_{o}}{\sqrt{\varepsilon_{o}} \sqrt{\varepsilon_{p}}} \int_{S_{m}} d \boldsymbol{r} \boldsymbol{t}_{m}(\boldsymbol{r}) \cdot \boldsymbol{H}^{\mathrm{inc}}(\boldsymbol{r}) .
\end{gathered}
$$

In the above, $\boldsymbol{b}_{n}$ and $\boldsymbol{t}_{m}$ are the basis and testing functions for $\{n, m\}=\{1,2, \ldots, N\}$, and $P V$ in Eq. (3) represents the principal value of the integral. In the numerical solutions in this chapter, we consider the Rao-Wilton-Glisson (RWG) functions defined on pairs of discretization triangles, that is, $\boldsymbol{b}_{n}(\boldsymbol{r})=0$ if $\boldsymbol{r} \notin S_{n}$ and $\boldsymbol{t}_{m}(\boldsymbol{r})=0$ if $\boldsymbol{r} \notin S_{m}$. Solution of the matrix equation in Eq. (1) provides the coefficients expanding the current densities, that is, $\boldsymbol{a}_{J}$ and $\boldsymbol{a}_{M}$ in $\boldsymbol{J}(\boldsymbol{r})=\sum \boldsymbol{a}_{J}[n] \boldsymbol{b}_{n}(\boldsymbol{r})$ and $\boldsymbol{M}(\boldsymbol{r})=\sum \boldsymbol{a}_{M}[n] \boldsymbol{b}_{n}(\boldsymbol{r})$.

It is remarkable that MCTF is stable for arbitrarily large values of $\varepsilon_{R}$ (as opposed to the conventional formulations [37]). As $\varepsilon_{R} \rightarrow \infty$ and $\varepsilon_{p} \rightarrow-\infty$, we have $\boldsymbol{a}_{M} \rightarrow 0$, which is enforced by the second row that reduces into

$$
\bar{G} \cdot a_{M}=0
$$

In the above, $\bar{G}$ is the Gram matrix, that is,

$$
\bar{G}[m, n]=\int_{S_{m}} d \boldsymbol{r t}_{m}(\boldsymbol{r}) \cdot \boldsymbol{b}_{n}(\boldsymbol{r}),
$$

which contains only five nonzero elements (corresponding to the intersections of the RWG functions) per row or column. While the second row leads to vanishing magnetic currents, the first row of MCTF becomes 


$$
i \omega \mu_{o} \int_{S_{m}} d \boldsymbol{r} \boldsymbol{t}_{m}(\boldsymbol{r}) \cdot \sum_{n=1}^{N} \boldsymbol{a}_{J}[n] \int_{S_{n}} d \boldsymbol{r}^{\prime} g_{o}\left(\boldsymbol{r}, \boldsymbol{r}^{\prime}\right) \boldsymbol{b}_{n}\left(\boldsymbol{r}^{\prime}\right)=-\int_{S_{m}} d \boldsymbol{r} \boldsymbol{t}_{m}(\boldsymbol{r}) \cdot \boldsymbol{E}^{\mathrm{inc}}(\boldsymbol{r})
$$

for $m=1,2, \ldots, N$, which is the standard electric-field integral equation for perfectly conducting objects.

\subsection{Accelerated matrix-vector multiplications with MLFMA}

Matrix equations derived from MCTF can be solved iteratively, where the matrix-vector multiplications can be performed efficiently as

$$
\left[\begin{array}{l}
\boldsymbol{y}_{1} \\
\boldsymbol{y}_{2}
\end{array}\right]=\left[\begin{array}{ll}
\overline{\boldsymbol{Y}}_{11}^{\mathrm{MCTF}} & \overline{\boldsymbol{Y}}_{12}^{\mathrm{MCTF}} \\
\overline{\boldsymbol{Y}}_{21}^{\mathrm{MCTF}} & \overline{\boldsymbol{Y}}_{22}^{\mathrm{MCTF}}
\end{array}\right] \cdot\left[\begin{array}{c}
\boldsymbol{x}_{J} \\
\boldsymbol{x}_{M}
\end{array}\right]+\left[\begin{array}{ll}
\overline{\boldsymbol{u}}_{11}^{\mathrm{MCTF}} & \overline{\boldsymbol{u}}_{12}^{\mathrm{MCTF}} \\
\overline{\boldsymbol{u}}_{21}^{\mathrm{MCTF}} & \overline{\boldsymbol{u}}_{22}^{\mathrm{MCTF}}
\end{array}\right] \cdot\left[\begin{array}{c}
\boldsymbol{x}_{J} \\
\boldsymbol{x}_{M}
\end{array}\right],
$$

where $\bar{Y}_{a b}^{\mathrm{MCTF}}$ for $\{a, b\}=\{1,2\}$ represents the near-field interactions that are between the basis and testing functions close to each other for a given distance threshold. There are only $\mathcal{O}(N)$ nonzero elements in each $\bar{Y}_{a b}^{\mathrm{MCTF}}$ with an identical sparsity pattern. Most of the interactions (computations in matrix-vector multiplications) corresponding to the elements in the far-field matrices $\overline{\boldsymbol{U}}_{a b}^{\mathrm{MCTF}} \approx \overline{\boldsymbol{Z}}_{a b}^{\mathrm{MCTF}}-\overline{\boldsymbol{Y}}_{a b}^{\mathrm{MCTF}}$ are calculated on the fly using MLFMA. These matrix elements are never computed directly or stored in memory, while their multiplications with given input vectors $\boldsymbol{x}_{J}$ and $\boldsymbol{x}_{M}$ can still be performed with controllable accuracy, as described in [36]. Using MLFMA, a matrix equation can efficiently be solved via Krylov-subspace algorithms with $\mathcal{O}(N \log N)$ complexity per matrix-vector multiplication or iteration.

In the solutions of plasmonic problems, inner interactions are localized as the negative permittivity increases. Since MCTF involves the combinations of interactions from the inner and outer media, the contributions from the inner medium become very small for some longdistance interactions. This allows us to omit such contributions without deteriorating the accuracy [38]. As an example, consider the first term for $\bar{Z}_{11}^{\mathrm{MCTF}}$ in Eq. (2). The integrand involves the combination of Green's functions as

$$
g_{o}\left(\boldsymbol{r}, \boldsymbol{r}^{\prime}\right)+g_{p}\left(\boldsymbol{r}, \boldsymbol{r}^{\prime}\right)=\frac{\exp \left(i k_{o}\left|\boldsymbol{r}-\boldsymbol{r}^{\prime}\right|\right)}{4 \pi\left|\boldsymbol{r}-\boldsymbol{r}^{\prime}\right|}-\frac{\exp \left(i k_{p}\left|\boldsymbol{r}-\boldsymbol{r}^{\prime}\right|\right)}{4 \pi\left|\boldsymbol{r}-\boldsymbol{r}^{\prime}\right|}=\frac{\exp \left(i k_{o}\left|\boldsymbol{r}-\boldsymbol{r}^{\prime}\right|\right)+\exp \left(i k_{p}\left|\boldsymbol{r}-\boldsymbol{r}^{\prime}\right|\right)}{4 \pi\left|\boldsymbol{r}-\boldsymbol{r}^{\prime}\right|} .
$$

In a normalized form, the contribution of the inner medium becomes more obvious as

$$
\frac{g_{o}\left(\boldsymbol{r}, \boldsymbol{r}^{\prime}\right)+g_{p}\left(\boldsymbol{r}, \boldsymbol{r}^{\prime}\right)}{g_{o}\left(\boldsymbol{r}, \boldsymbol{r}^{\prime}\right)}=1+\frac{\exp \left(i k_{p}\left|\boldsymbol{r}-\boldsymbol{r}^{\prime}\right|\right)}{\exp \left(i k_{o}\left|\boldsymbol{r}-\boldsymbol{r}^{\prime}\right|\right)} .
$$

If the interactions in MLFMA are to be calculated with an error threshold of $\Delta e$, which is determined by the sampling and truncation used in the diagonalization of Green's function [34], it is possible to omit the contribution from the inner medium if 


$$
\left|\exp \left(i k_{p}\left|\boldsymbol{r}-\boldsymbol{r}^{\prime}\right|\right)\right| \leq \Delta e\left|\exp \left(i k_{o}\left|\boldsymbol{r}-\boldsymbol{r}^{\prime}\right|\right)\right|=\Delta e
$$

For large values of $\varepsilon_{R}$, this inequality can be approximated as

$$
\left|\boldsymbol{r}-\boldsymbol{r}^{\prime}\right| \geq \frac{-\ln (\Delta e)}{k_{o} \sqrt{\varepsilon_{R}}}
$$

as the condition for the distance to omit the related inner interaction. For example, for two digits of accuracy $(\Delta e=0.01)$ and $\varepsilon_{R}=100$ at $250 \mathrm{THz}$, it is safe to omit the interaction if $\left|\boldsymbol{r}-\boldsymbol{r}^{\prime}\right| \geq 88 \mathrm{~nm}$. This significantly accelerates the matrix-vector multiplications with MLFMA. We emphasize that omitting interactions as described above has no negative effect in the accuracy of the results. In fact, as the frequency drops and $\varepsilon_{R}$ increases, we expect that inner interactions decay quickly such that the inner matrix becomes a Gram matrix and the formulation turns into a perfectly conducting version.

\section{Nanowire simulations}

In this section, we present the major parameters of the nanowire simulations considered in this chapter using the implementation described in Section 2.

\subsection{Nanowire models}

We consider Ag nanowires of length $5 \mu \mathrm{m}$ at a fixed frequency of $250 \mathrm{THz}$, at which the nanowire length corresponds to approximately $4.17 \lambda_{0}$. At this frequency, the permittivity of $\mathrm{Ag}$ can be found as approximately $-60.76+4.31 i$ [20]. For the cross sections of the nanowires, we use square, circular (circular-like, more precisely, depending on the discretization size), and hexagonal shapes. For all shapes, the cross-sectional area of each nanowire is selected as $0.01 \mu \mathrm{m}^{2}$ (e.g., a square nanowire has $0.1 \times 0.1 \mu \mathrm{m}$ cross section) other than scaling trials for the hexagonal cases. For numerical solutions, nanowires are discretized with $0.05-0.075 \mu \mathrm{m}$ triangles $\left(\lambda_{0} / 24-\lambda_{0} / 16\right)$, on which the RWG functions are defined. As shown in the numerical results, we consider arrays of nanowires in $2 \times 1,2 \times 2,4 \times 4$, and $6 \times 6$ arrangements, while the main focus is the comparison of the $6 \times 6$ arrays. In the regular arrays, the distance between the centers of two nanowires is $0.2 \mu \mathrm{m}$. In the hexagonal arrangements involving hexagonal cells, the center-tocenter distance between two nearby nanowires is approximately $0.186 \mu \mathrm{m}$. Discretization of each nanowire requires $2772-5376$ unknowns. Then, for the $6 \times 6$ arrays, these discretizations lead to matrix equations involving 99,792-193,536 unknowns, which need a fast and efficient solver, such as MLFMA, to complete all simulations in hours.

\subsection{Excitation}

Each nanowire array is excited by a pair of Hertzian dipoles oriented in the opposite directions. The electric and magnetic field intensities created by a single dipole can be written as 


$$
\begin{gathered}
\boldsymbol{E}^{\mathrm{inc}}(\boldsymbol{r})=i \omega \mu_{0} \frac{\exp \left(i k_{o} R\right)}{4 \pi R}\left\{\boldsymbol{I}_{D}\left(1+\frac{i}{k_{0} R}-\frac{1}{k_{o}^{2} R^{2}}\right)-\boldsymbol{I}_{D} \cdot \hat{\boldsymbol{R}} \hat{\boldsymbol{R}}\left(1+\frac{3 i}{k_{o} R}-\frac{3}{k_{o}^{2} R^{2}}\right)\right\} \\
\boldsymbol{H}^{\mathrm{inc}}(\boldsymbol{r})=\boldsymbol{I}_{D} \times \hat{\boldsymbol{R}} \frac{\exp \left(i k_{o} R\right)}{4 \pi R}\left(\frac{1}{R}-i k_{o}\right),
\end{gathered}
$$

where $\boldsymbol{R}=\boldsymbol{r}-\boldsymbol{r}^{\prime}=\hat{\boldsymbol{R}} R$ and $\boldsymbol{I}_{D}$ is the dipole moment. The distance between the dipoles is 0.2 $\mu \mathrm{m}$ and they are located symmetrically with $0.2 \mu \mathrm{m}$ distance from the nanowires. An example to the localization of the dipoles with respect to a $4 \times 4$ array of square nanowires is depicted in Figure 1. Except for the $2 \times 1$ arrays, the transverse locations of the dipoles correspond to gaps between the nanowires, leading to efficient dipole-to-array coupling. For the same reason, the power flow is mainly confined inside these arrays (on the interfaces between the nanowires and gaps), while for the $2 \times 1$ arrays (and somewhat for the $2 \times 2$ arrays), the transmission occurs mostly on the outer sides.

\subsection{Field and power calculations}

After the current coefficients are found through iterative solutions employing MLFMA, the near-zone electric and magnetic fields can be found anywhere using the discretized form of

$$
\begin{gathered}
\boldsymbol{E}^{\mathrm{sec}}(\boldsymbol{r})=i \omega \mu_{o} \int \boldsymbol{d} \boldsymbol{r}^{\prime} \boldsymbol{J}\left(\boldsymbol{r}^{\prime}\right) g_{u}\left(\boldsymbol{r}, \boldsymbol{r}^{\prime}\right)+\frac{i}{\omega \varepsilon_{u}} \int d \boldsymbol{r}^{\prime} \nabla^{\prime} \cdot \boldsymbol{J}\left(\boldsymbol{r}^{\prime}\right) \nabla g_{u}\left(\boldsymbol{r}, \boldsymbol{r}^{\prime}\right)-\int d r^{\prime} \nabla g_{u}\left(\boldsymbol{r}, \boldsymbol{r}^{\prime}\right) \times \boldsymbol{M}\left(\boldsymbol{r}^{\prime}\right) \\
\boldsymbol{H}^{\mathrm{sec}}(\boldsymbol{r})=i \omega \varepsilon_{u} \int d \boldsymbol{r}^{\prime} \boldsymbol{M}\left(\boldsymbol{r}^{\prime}\right) g_{u}\left(\boldsymbol{r}, \boldsymbol{r}^{\prime}\right)+\frac{i}{\omega \mu_{o}} \int d \boldsymbol{r}^{\prime} \nabla^{\prime} \cdot \boldsymbol{M}\left(\boldsymbol{r}^{\prime}\right) \nabla g_{u}\left(\boldsymbol{r}, \boldsymbol{r}^{\prime}\right)+\int d \boldsymbol{r}^{\prime} \boldsymbol{J}\left(\boldsymbol{r}^{\prime}\right) \times \nabla^{\prime} g_{u}\left(\boldsymbol{r}, \boldsymbol{r}^{\prime}\right),
\end{gathered}
$$

where the integrals are over the surfaces of the structure. The calculations are carried out for the outer $(u=o)$ and inner $(u=p)$ problems, leading to vanishingly zero fields for the inner and outer regions, respectively. This also allows us to check the accuracy of solutions by testing the equivalence theorem. We note that, for the outer problem, incident fields $\boldsymbol{E}^{\text {inc }}$ and $\boldsymbol{H}^{\text {inc }}$ must be added to the secondary fields $\boldsymbol{E}^{\mathrm{sec}}$ and $\boldsymbol{H}^{\mathrm{sec}}$ to arrive at the total values. Then, by superimposing the results from inner and outer calculations, overall graphics demonstrating the electric and magnetic field intensities in the vicinity of the object are obtained. We

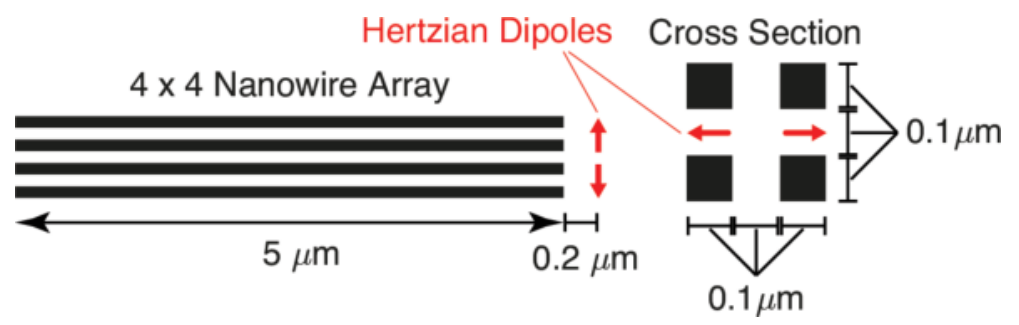

Figure 1. Positions of Hertzian dipoles with respect to a $4 \times 4$ nanowire array. The length of all arrays considered in this chapter is $5 \mu \mathrm{m}$, while the dipoles oriented in the opposite directions are located symmetrically with $0.2 \mu \mathrm{m}$ distance from the nanowires. 
particularly focus on the magnitudes of the electric field intensity and the magnetic field intensity with units $\mathrm{V} / \mathrm{m}$ and $\mathrm{A} / \mathrm{m}$, respectively, in $\mathrm{dB}$ scales. In addition, as a major quantity in the analysis of nanowires, we calculate the Poynting vector $\left(\mathrm{W} / \mathrm{m}^{2}\right)$, simply called the power density in this chapter, as

$$
\boldsymbol{S}(\boldsymbol{r})=\frac{1}{2} \boldsymbol{E}(\boldsymbol{r}) \times \boldsymbol{H}^{*}(\boldsymbol{r}),
$$

where $^{*}$ is the conjugate operation. In this equation, $\boldsymbol{E}$ and $\boldsymbol{H}$ represent the field intensities, corresponding to only secondary fields inside the object and the sum of secondary and incident fields outside the object. We note that the time-average power density is the real part of the expression in Eq. (20). Similar to the field intensities, we present the magnitude of the power density in the results. Considering the continuity of the tangential fields on surfaces, the normal component of the power density is continuous across nanowire/air interfaces, while the main power flow is in the tangential directions (along nanowires). As an example to field intensity and power density distributions, Figure 2 depicts the excitation of a pair of nanowires with square cross sections. Electric field intensity, magnetic fields intensity, and the real part of the power density are plotted in the vicinity of the dipoles and the tips of the nanowires, where the coupling of the electromagnetic energy to the nanowire system is clearly visible.

\subsection{Iterative solutions and parameters}

Nanowire problems are challenging in terms of iterative solutions, partially due to MCTF that provides accurate solutions of plasmonic problems at the cost of worse conditioning of matrix equations in comparison to those derived from the second-kind integral equations. Therefore, iterative solutions are performed using a flexible generalized minimal residual (FGMRES) algorithm, which allows for a nested strategy involving inner solutions through GMRES. Specifically, FGMRES employs MLFMA (with 1\% maximum error in all solutions) for the matrix-vector multiplications, while it is preconditioned by inner solutions via GMRES. In the inner layer, GMRES employs an approximate form of MLFMA (AMLFMA) [39] that is derived from MLFMA by reducing the number of harmonics. GMRES is further preconditioned via
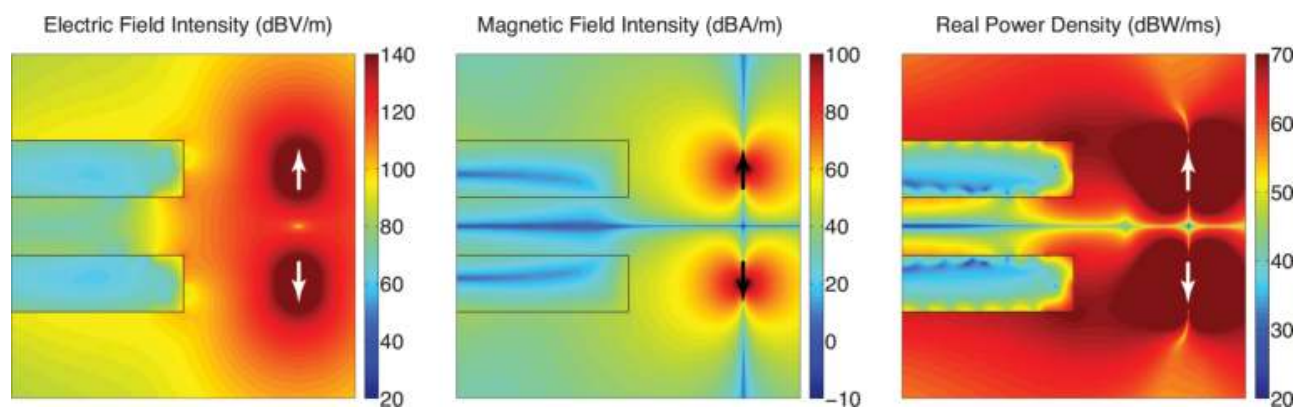

Figure 2. Excitation of a pair of nanowires with square cross sections through a pair of Hertzian dipoles. The electric field intensity, magnetic field intensity, and the real part of the power density are plotted in the vicinity of the dipoles (shown with arrows) and the tips of the nanowires. 

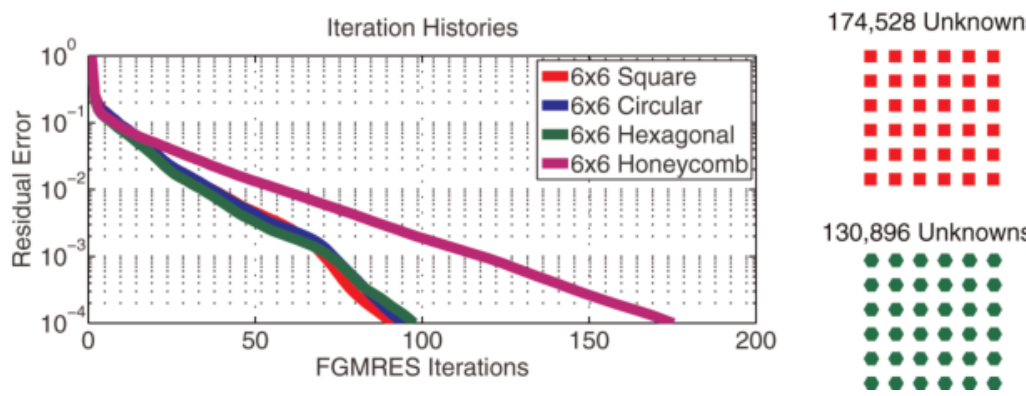

193,536 Unknowns

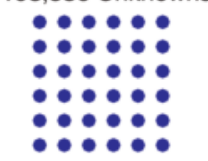

130,896 Unknowns

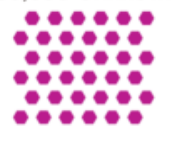

Figure 3. Iteration histories for the solutions of $6 \times 6$ nanowire arrays.

algebraic preconditioners based on the near-field interactions. As an example to iterative solutions, Figure 3 depicts the iteration histories for the analysis of $6 \times 6$ nanowire arrays (with number of unknowns shown in Figure 3). The residual error is reduced to $10^{-4}$ (as in all solutions presented in this chapter), requiring nearly 100 iterations for the circular, square, and hexagonal cross sections. For the honeycomb structure, the number of iterations increases to more than 150 to achieve the desired residual error. The reason for larger number of iterations for the honeycomb structure seems to be related to very high transmission capabilities of the nanowires in this configuration, as shown in the simulation results.

\section{Nanowire arrays for power transfer}

Using the developed solver, we consider nanowires and their arrangements in alternative configurations. Near-zone field intensity and power density values, which are computed accurately with given error thresholds as described above, are used to precisely assess the efficiency of power transmission levels, leading to a rigorous study of nanowire arrays as transmission lines at optical frequencies.

\subsection{Double nanowires}

First, we consider transmission by pairs of nanowires with different cross sections. Figure 4 presents the electric field intensity $(\mathrm{dBV} / \mathrm{m})$, magnetic field intensity $(\mathrm{dBA} / \mathrm{m})$, and power density $\left(\mathrm{dBW} / \mathrm{m}^{2}\right)$ in the vicinity of the nanowires (on a plane containing dipoles). In each case, a total of $200 \times 800=160,000$ samples are used for high-quality plots. The excitation dipoles are located on the right-hand side, as clearly observed by large intensity and density values on this side. We observe that the power transmission is almost identical for different cross sections, that is, circular, hexagonal, and square. We note that the nanowires have the same cross-sectional area and the distance between them is the same for all cases. In general, there is a high-quality transmission of the electromagnetic energy along the nanowires, independent of the cross section. In each case, the variations in the electric field intensity, magnetic field intensity, and power density are related to the optical length of the nanowires, which is $5 \mu \mathrm{m}$ corresponding to approximately $4.17 \lambda_{0}$. While not clearly visible in these plots, 


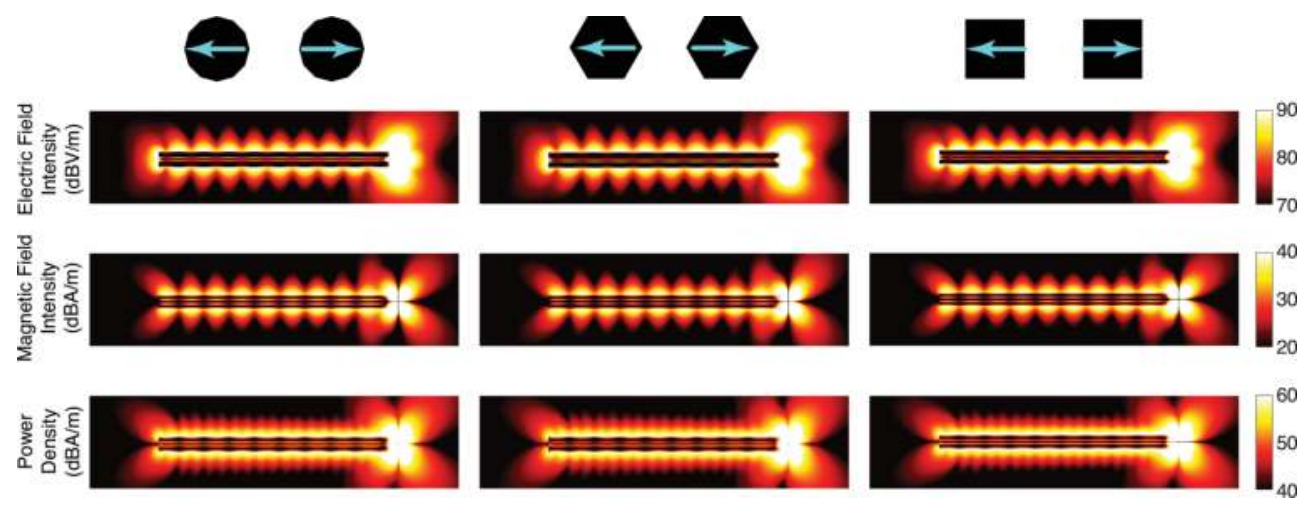

Figure 4. The electric field intensity, magnetic field intensity, and power density values in the vicinity of $2 \times 1$ nanowire arrays with different cross sections. The transverse locations of the dipoles (arrows) are also shown.

plasmonic oscillations with much higher rates in the power values exist on the air/metal interfaces, as depicted in Figure 2 for the nanowires with square cross sections. Figure 4 also shows that when the transmission line involves only two nanowires, the transmission occurs mostly along the outer surfaces.

\subsection{Nanowires in array configurations}

Next, we focus on $2 \times 2$ systems of nanowires, as depicted in Figure 5, again with different cross sections. In this case, the near-field samples (that are on the plane containing the dipoles) do not coincide with the nanowires. Comparing the results for different cross sections, we observe similar characteristics with high-quality transmissions. Specifically, none of the crosssectional shapes seems to have an advantage over others. Considering the outputs of the
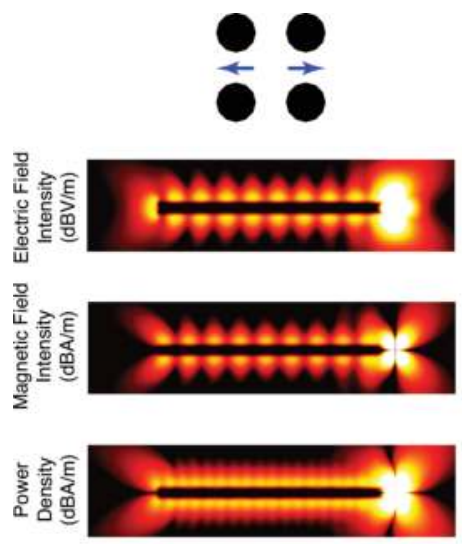
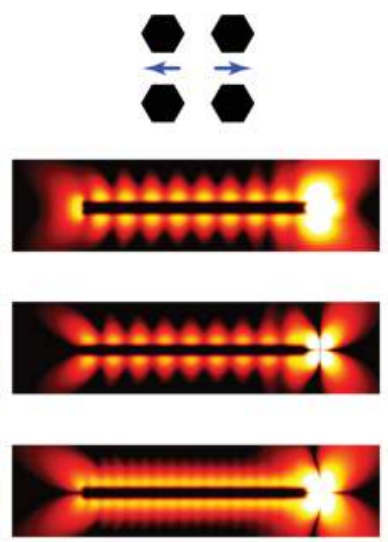
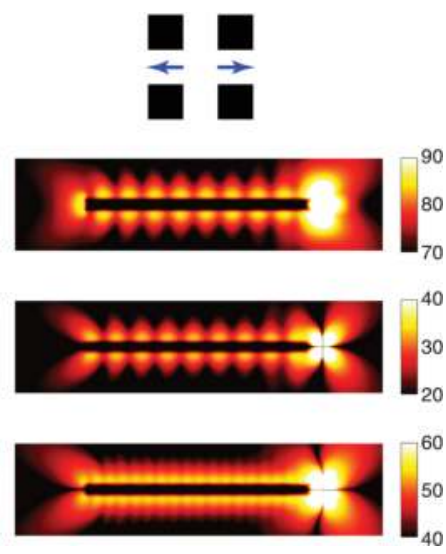

Figure 5. The electric field intensity, magnetic field intensity, and power density values in the vicinity of $2 \times 2$ nanowire arrays with different cross sections. The transverse locations of the dipoles (arrows) are also shown. 
transmission lines, where the electromagnetic energy is coupled to air, $2 \times 2$ arrays seem to be slightly better than the nanowire pairs shown in Figure 4. For example, considering the power density distributions, larger beams occur on the left-hand sides of the plots, indicating better transmissions with the $2 \times 2$ arrays. Values along the nanowires seem to decrease in comparison to $2 \times 1$ systems, but this is (misleadingly) due to the sampling plane that is separated from the nanowires for the $2 \times 2$ arrangements. Therefore, for a fair comparison, we need to consider the outputs of the nanowires (on the left-hand sides of the plots).

Figure 6 presents similar results for $4 \times 4$ arrays. In this case, in addition to circular, square, and hexagonal nanowires that are regularly arranged, we consider a small-scale honeycomb structure involving hexagonal nanowires in a hexagonal arrangement. We note that the term honeycomb is used to describe the overall structure with hexagonal gaps between hexagonal nanowires with the same cross-sectional areas. In comparison to smaller arrays, the transmissions through $4 \times 4$ arrays, which occur in two parallel paths for the regular arrangements, are mostly confined inside the arrays. It is remarkable that the transmission properties are almost the same for the regular arrangements of the nanowires with circular, square, and hexagonal cross sections, while the transmission along the honeycomb structure occurs differently as distributed into more pathways. Nevertheless, considering again the outputs of the nanowires, there is not a clear advantage of this structure over others.

\subsection{Comparisons of larger arrays}

Dramatic changes occur when the nanowire arrays get larger. In Figure 7, we consider arrays of $6 \times 6$ nanowires that are arranged similar to the $4 \times 4$ arrays. For the regular arrays of nanowires with circular, square, and hexagonal cross sections, the electromagnetic energy is transmitted again in two main lines close to the transverse locations of the dipoles. The transmission performance of these arrays is very similar to each other. On the other hand, a significant improvement is observed when the arrangement is also made hexagonal, leading to a honeycomb structure. This structure clearly outperforms the others with higher quality field and power transmissions. This is further verified in Figure 8, where the intensity and density values are sampled at the outputs of the arrays. In these plots, the samples are selected on the transverse plane located at $0.2 \mu \mathrm{m}$ distance from the nanowire tips (at the output side). In the power density values, more than $5 \mathrm{~dB}$ (more than three times) enhancement is observed when the honeycomb structure is used instead of the others.

\subsection{Investigation of cross-sectional area}

After demonstrating the significantly higher transmission capabilities of the honeycomb structure, it is questioned whether the cross-sectional areas of the nanowires are optimal or not. For this purpose, we consider hexagonal arrangements of $6 \times 6$ nanowires with only hexagonal cross sections. As depicted in Figure 9, we scale the cross-sectional dimensions by 0.5, 0.75, 1.25 , and 1.375 , when 1.0 corresponds to the original honeycomb structure. It can be observed that scaling cross-sectional dimensions both to smaller and to larger values leads to deteriorations in the transmission properties of the array. This is again verified by the output plots in Figure 10, where the results for a scaling with 1.125 ratio is included as well as for the original 


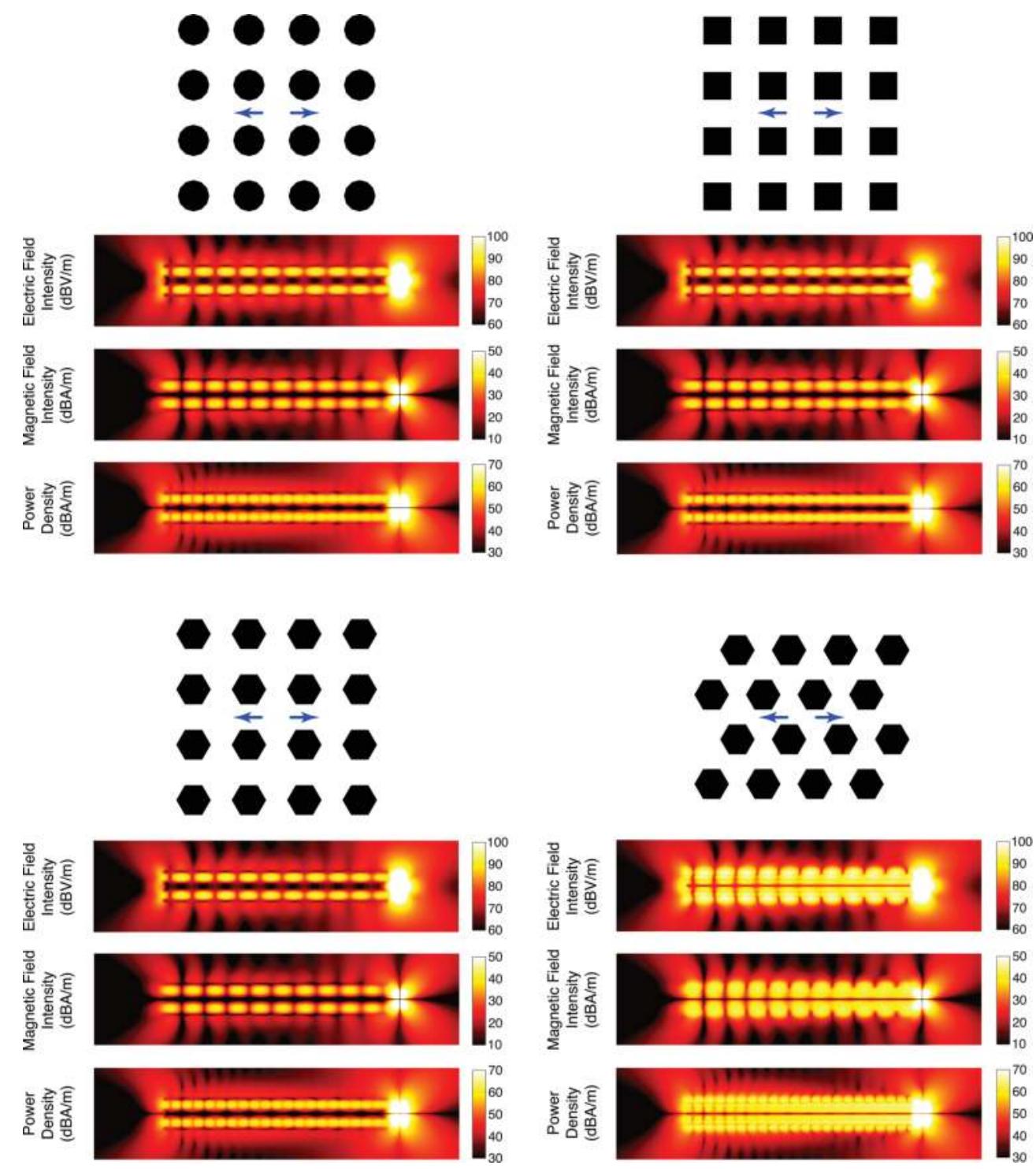

Figure 6. The electric field intensity, magnetic field intensity, and power density values in the vicinity of $4 \times 4$ nanowire arrays with different cross sections. The transverse locations of the dipoles (arrows) are also shown. We note that, in the longitudinal direction, the distance between the dipoles and the arrays is $0.2 \mu \mathrm{m}$ in all cases. 

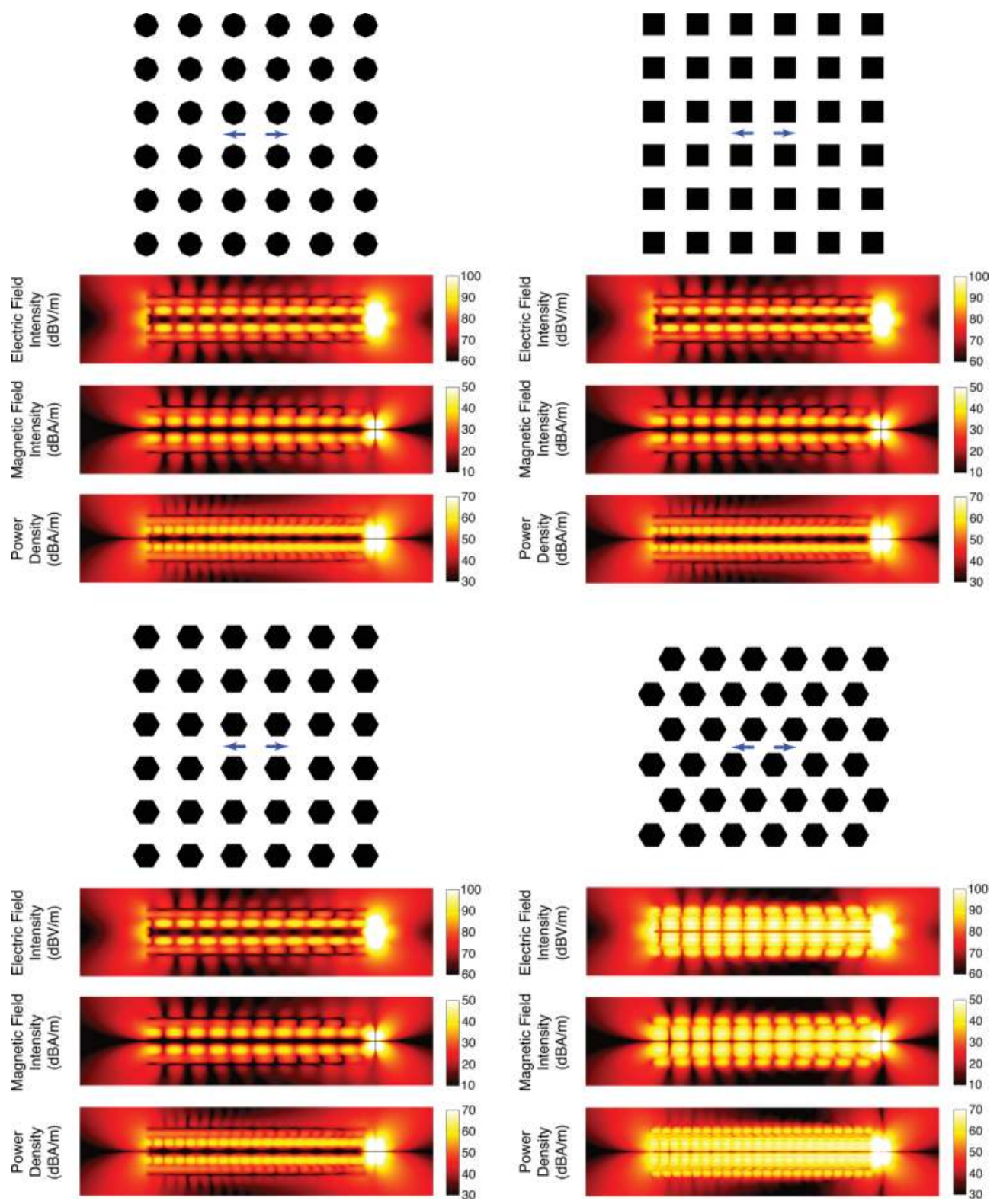

Figure 7. The electric field intensity, magnetic field intensity, and power density values in the vicinity of $6 \times 6$ nanowire arrays with different cross sections. The transverse locations of the dipoles (arrows) are also shown. We note that, in the longitudinal direction, the distance between the dipoles and the arrays is $0.2 \mu \mathrm{m}$ in all cases. 

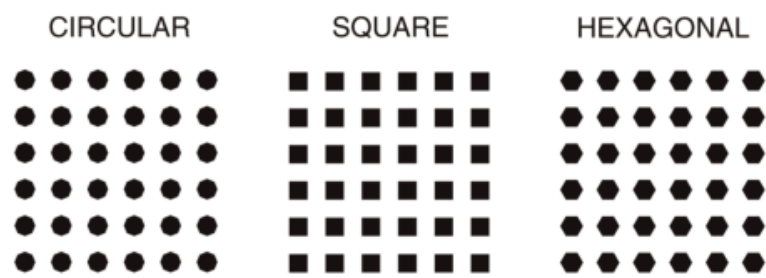

HONEYCOMB
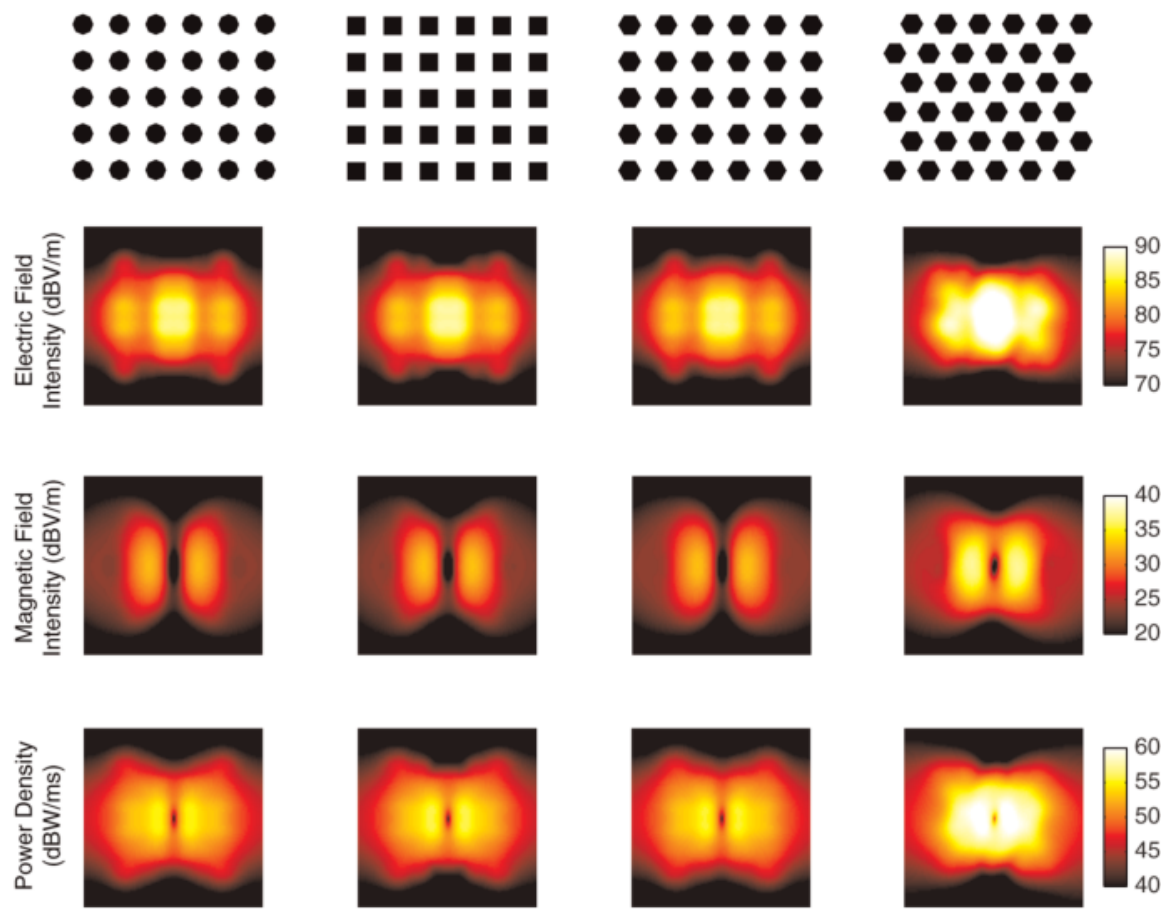

Figure 8. The electric field intensity, magnetic field intensity, and power density values at the outputs of $6 \times 6$ nanowire arrays with different cross sections.

structure. As demonstrated in these plots, even small changes in the cross-sectional areas of the nanowires deteriorate the performance of the array, showing the optimality of the honeycomb structure with equally filled and empty spaces.

\section{Discussion}

Based on the numerical results in Section 4, one may conclude that

- the $6 \times 6$ honeycomb structure demonstrates very good transmission properties, in comparison to other arrays with the same number of nanowires under the same conditions,

- closely packing the hexagonal nanowires (scaling up) deteriorates the transmission quality, and

- $\quad$ reducing the dimensions (scaling down) also has a negative effect.

In order to explain the results, we now consider the electric current density $(\boldsymbol{J}=\hat{\boldsymbol{n}} \times \boldsymbol{H})$ on the nanowire surfaces. Figure $\mathbf{1 1}$ depicts the dominant electric current (in dB scale with $20 \mathrm{~dB}$ 

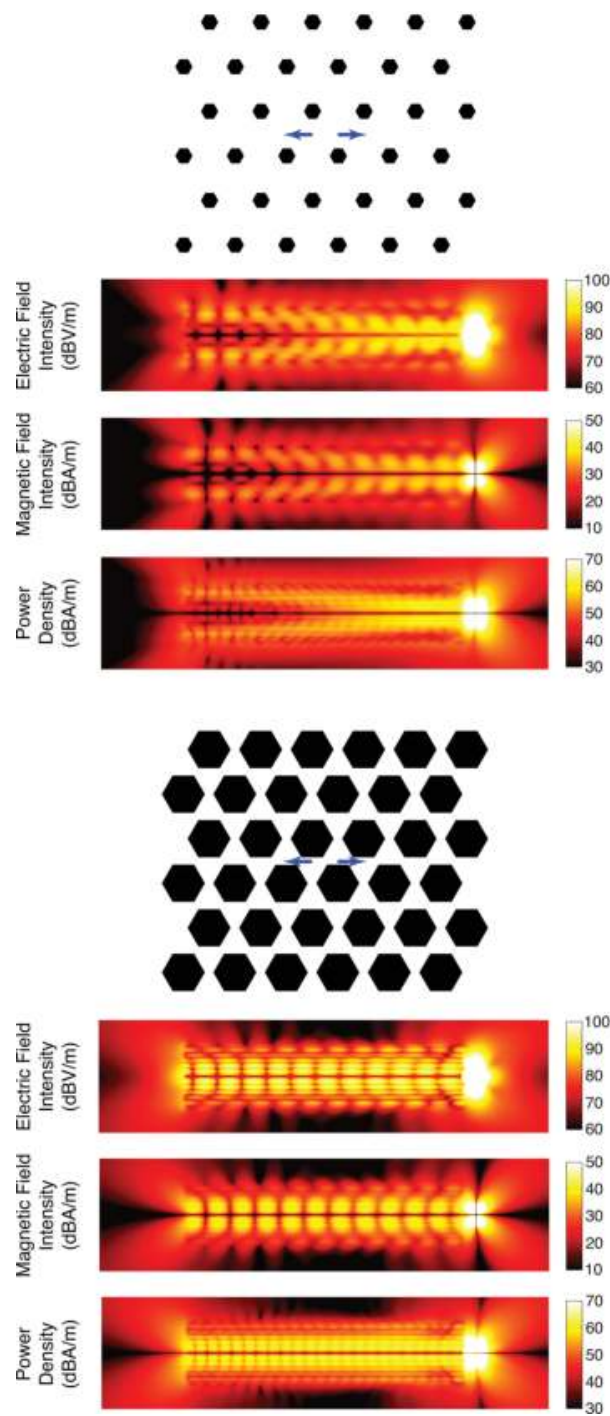
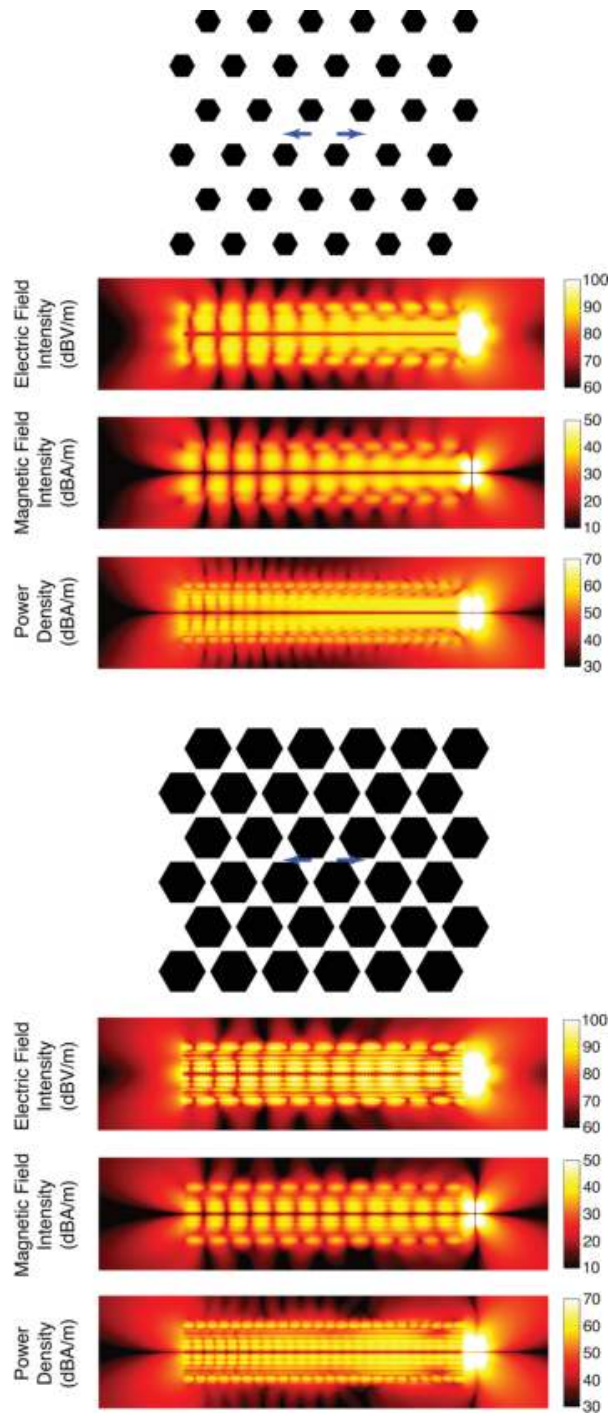

Figure 9. The electric field intensity, magnetic field intensity, and power density values in the vicinity of $6 \times 6$ nanowire arrays with hexagonal cross sections of different areas. We note that, in the longitudinal direction, the distance between the dipoles and the arrays is $0.2 \mu \mathrm{m}$ in all cases.

dynamic range) along the $6 \times 6$ arrays of nanowires with circular, square, and hexagonal cross sections, as well as along the honeycomb structure. We also consider the hexagonal arrangement of hexagonal nanowires scaled by the factors of 0.5 and 1.4. It is evident that the current density is much higher for the honeycomb structure. In addition to three-dimensional views, we present a side view when the dipoles are oriented outward/inward. Comparison of current distributions reveals significant differences on the behaviors of the arrays. First, considering 


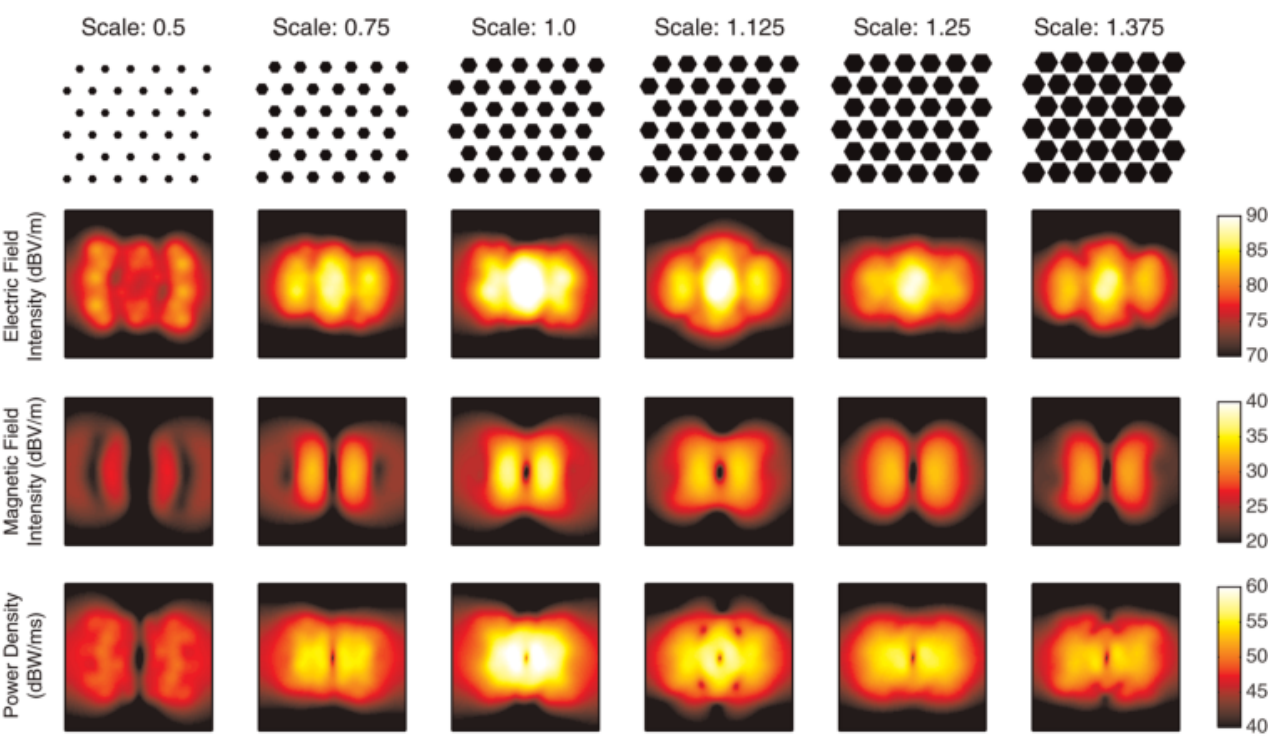

Figure 10. The electric field intensity, magnetic field intensity, and power density values at the outputs of $6 \times 6$ nanowire arrays with hexagonal cross sections of different areas. The scale of 1.0 corresponds to the original honeycomb structure.

the array with circular cross sections, it can be observed that the currents along the nanowires are out of phase. Specifically, the current density has maximum locations at different longitudinal positions for the inner two rows and the outer rows. The same situation is also observed for the arrays with square and hexagonal cross sections. All these observations are consistent with the near-zone plots depicted in Figure 7.

The out-of-phase currents along the regular arrays can be explained by the adverse effects of neighboring nanowires. Specifically, considering Ampere's circuital law, each nanowire creates a magnetic field that induces current in the opposite direction (to the main current flow) on the neighboring nanowires. Due to the cancellations of the main and secondary currents, the overall currents along the nanowires become out of phase, reducing the transmission capability of the arrays. From this perspective, nanowires in a honeycomb structure are located optimally such that the adverse interactions between nanowires are minimized. Considering how the nanowires are located, it can be observed that nearby surfaces of neighboring nanowires are not aligned and the magnetic coupling between the nanowires is weaker than those in the other arrangements. There are surfaces with identical alignments but these surfaces (on the same sides of the nanowires) are far away from each other. As a result, the currents along the nanowires are almost in phase, as depicted in Figure $\mathbf{1 1 .}$

Scaling the nanowires in the honeycomb structures always has an adverse effect. Decreasing cross-sectional area reduces the overall area of the surfaces for the propagation of plasmonic waves. In addition, increasing distance between the surfaces of the neighboring nanowires adds extra phase in the magnetic coupling, deteriorating the synchronization of the currents. Obviously, increasing the cross-sectional area also has adverse effect as a result of increasing 

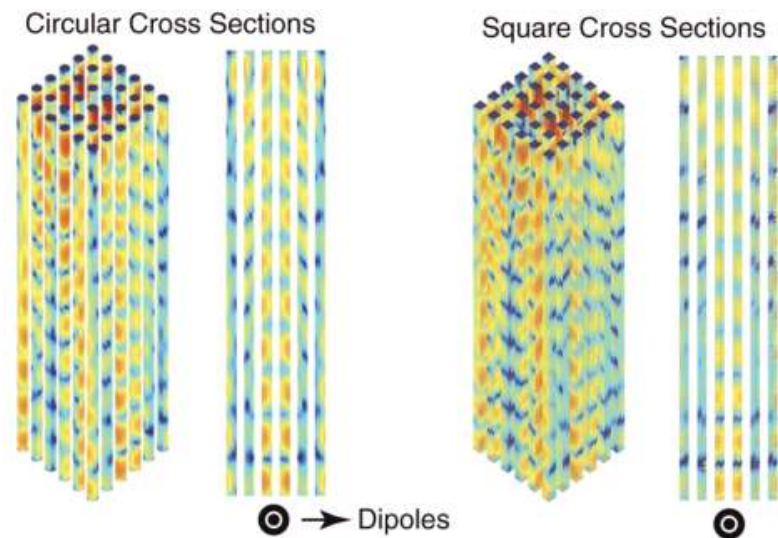

$\circ$

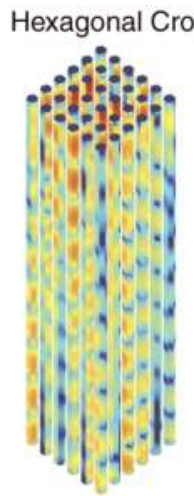

Honeycomb Structure

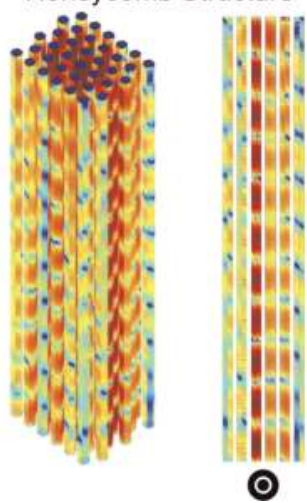

Scaled By 0.5

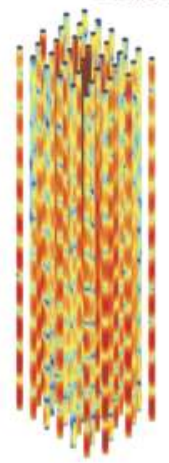

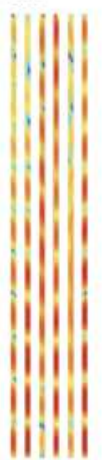

○

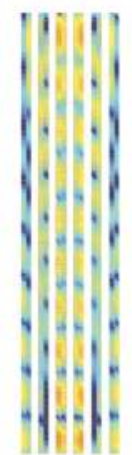

()
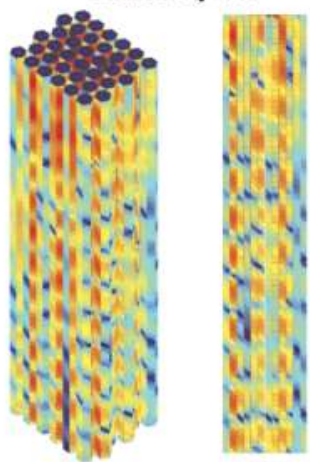

○

Figure 11. The magnitude of the electric current density induced on the nanowire surfaces for different array configurations. The location and orientation (outward and inward) of the dipole pair are shown in side views.

coupling between the nanowires. It appears that the honeycomb structure is a kind of optimal way to transmit power.

\section{Concluding remarks}

An investigation of nanowire transmission lines may lead to optimal structures that can be used in nano-optical devices. In this chapter, we present the effects of cross-sectional geometry and arrangement of array elements in different configurations. Using an accurate full-wave simulation environment, we obtain reliable results of three-dimensional finite arrays without resorting to approximation methods and asymptotic optical techniques that may have significant accuracy problems. Based on a comparative study of $\mathrm{Ag}$ nanowires at an infrared frequency, we show that power transmission becomes significantly higher for nanowires with hexagonal cross sections and their hexagonal arrangements, leading to honeycomb structures. We also explain the favorable electromagnetic characteristics of these structures, which may pave a way for improved nanowire systems for optimal power transmission. 


\section{Acknowledgements}

This work was supported by the Scientific and Technical Research Council of Turkey (TUBITAK) under the Research Grant 113E129 and by the Turkish Academy of Sciences (TUBA) in the framework of the Young Scientist Award Program.

\section{Authors contributions}

Ö.E. developed the theory and formulations, B.K. and Ö.E. implemented the solvers, and H.A.Ş. conducted the numerical experiments.

\section{Author details}

Hasan Aykut Şatana, Barışcan Karaosmanoğlu and Özgür Ergül*

*Address all correspondence to: ozgur.ergul@eee.metu.edu.tr

Department of Electrical and Electronics Engineering, Middle East Technical University, Ankara, Turkey

\section{References}

[1] Ditlbacher H, Hohenau A, Wagner D, Kreibig U, Rogers M, Hofer F, Aussenegg FR, Krenn JR. Silver nanowires as surface plasmon resonators. Phys. Rev. Lett. 2005;95: 257403.

[2] Sanders AW, Routenberg DA, Wiley BJ, Xia Y, Dufresne ER, Reed MA. Observation of plasmon propagation, redirection, and fan-out in silver nanowires. Nano Lett. 2006;6: 1822-1826.

[3] Akimov AV, Mukherjee A, Yu CL, Chang DE, Zibrov AS, Hemmer PR, Park H, Lukin MD. Generation of single optical plasmons in metallic nanowires coupled to quantum dots. Nature. 2007;450:402-406.

[4] Guo X, Qiu M, Bao J, Wiley BJ, Yang Q, Zhang X, Ma Y, Yu H, Tong L. Direct coupling of plasmonic and photonic nanowires for hybrid nanophotonic components and circuits. Nano Lett. 2009;9:4515-4519.

[5] Wang W, Yang Q, Fan F, Xu H, Wang ZL. Light propagation in curved silver nanowire plasmonic waveguides. Nano Lett. 2011;11:1603-1608. 
[6] Li Z, Bao K, Fang Y, Guan Z, Halas NJ, Nordlander P, Xu H. Effects of a proximal substrate on plasmon propagation in silver nanowires. Phys. Rev. B. 2010;82:241402.

[7] Ma Y, Li X, Yu H, Tong L, Gu Y, Gong Q. Direct measurement of propagation losses in silver nanowires. Opt. Lett. 2010;35:1160-1162.

[8] Yao J, Liu Z, Liu Y, Wang Y, Sun C, Bartal G, Stacy AM, Zhang X. Optical negative refraction in bulk metamaterials of nanowires. Science. 2008;321:930.

[9] Liu Y, Bartal G, Zhang X. All-angle negative refraction and imaging in a bulk medium made of metallic nanowires in the visible region. Opt. Exp. 2008;16:15439-15448.

[10] Casse BDF, Lu WT, Huang YJ, Gultepe E, Menon L. Super-resolution imaging using a three-dimensional metamaterials nanolens. Appl. Phys. Lett. 2010;96:023114.

[11] Sun M, Zhang Z, Wang P, Li Q, Ma F, Xu H. Remotely excited Raman optical activity using chiral plasmon propagation in Ag nanowires. Light Sci Appl. 2013;2:112.

[12] Huang Y, Fang Y, Zhang Z, Zhu L, Sun M. Nanowire-supported plasmonic waveguide for remote excitation of surface-enhanced Raman scattering. Light Sci Appl. 2014;3:199.

[13] Wang X, Summers CJ, Wang ZL. Large-scale hexagonal-patterned growth of aligned ZnO nanorods for nano-optoelectronics and nano-sensor arrays. Nano Lett. 2004;4:423-426.

[14] Rockstuhl C, Fahr S, Lederer F. Absorption enhancement in solar cells by localized plasmon polaritons. J. Appl. Phys. 2008;104:123102.

[15] Bergin SM, Chen Y, Rathmell AR, Charbonneau P, Lib Z, Wiley BJ. The effect of nanowire length and diameter on the properties of transparent, conducting nanowire films. Nanoscale. 2012;4:1996-2004.

[16] Yin AJ, Li J, Jian W, Bennett AJ, Xu JM. Fabrication of highly ordered metallic nanowire arrays by electrodeposition. Appl. Phys. Lett. 2001;79:1039-1041.

[17] Wang RC, Liu CP, Huang JL. ZnO hexagonal arrays of nanowires grown on nanorods. Appl. Phys. Lett. 2005;86:251104.

[18] Kottmann JP, Martin OJF. Plasmon resonances of silver nanowires with a non-regular cross section. Phys. Rev. B. 2001;64:235402.

[19] Yılmaz A, Karaosmanoğlu B, Ergül Ö. Computational electromagnetic analysis of deformed nanowires using the multilevel fast multipole algorithm. Sci. Rep. 2015;5:8469.

[20] Johnson PB, Christy RW. Optical constants of the noble metals. Phys. Rev. B. 1972;6:43704379 .

[21] Ergül Ö. Solutions of large-scale electromagnetics problems involving dielectric objects with the parallel multilevel fast multipole algorithm. J. Opt. Soc. Am. A. 2011;28:2261-2268.

[22] Kottmann JP, Martin OJF. Influence of the cross section and the permittivity on the plasmon-resonance spectrum of silver nanowires. Appl. Phys. B. 2001;73,299-304. 
[23] Futamata M, Maruyama Y, Ishikawa M. Local electric field and scattering cross section of Ag nanoparticles under surface plasmon resonance by finite difference time domain method. J. Phys. Chem. B. 2003;107:7607-7617.

[24] Giannini V, Rodriguez-Oliveros R, Sanchez-Gil JA. Surface plasmon resonances of metallic nanostars/nanoflowers for surface-enhanced Raman scattering. Plasmonics. 2010;5:99-104.

[25] Hohenester U, Krenn J. Surface plasmon resonances of single and coupled metallic nanoparticles: a boundary integral method approach. Phys. Rev. B. 2005;72:195429.

[26] Sondergaard T. Modeling of plasmonic nanostructures: green's function integral equation methods. Phys. Stat. Sol. B. 2007;244:3448-3462.

[27] Kern AM, Martin OFJ. Surface integral formulation for 3D simulations of plasmonic and high permittivity nanostructures. J. Opt. Soc. Am. A. 2009;26:732-740.

[28] Gallinet B, Martin OFJ. Scattering on plasmonic nanostructures arrays modeled with a surface integral formulation. Photon. Nanostruct. Fund. Appl. 2010;8:278-284.

[29] Rodriguez-Oliveros R, Sanchez-Gil JA. Localized surface-plasmon resonances on single and coupled nanoparticles through surface integral equations for flexible surfaces. Opt. Exp. 2011;16:12208-12219.

[30] Solis DM, Taboada JM, Obelleiro F. Surface integral equation method of moments with multiregion basis functions applied to plasmonics. IEEE Trans. Antennas Propag. 2015;63:2141-2152.

[31] Karaosmanoğlu B, Gür UM, Ergül Ö. Investigation of nanoantennas using surface integral equations and the multilevel fast multipole algorithm. In Proceedings of the Progress in Electromagnetics Research Symposium (PIERS); Prague, 2015, pp. 2026-2030.

[32] Bora M, Fasenfest BJ, Behymer EM, Chang ASP, Nguyen HT, Britten JA, Larson CC, Chan JW, Miles RR, Bond TC. Plasmon resonant cavities in vertical nanowire arrays. Nano Lett. 2010;10:2832-2837.

[33] Nauert S, Paul A, Zhen Y, Solis D, Vigderman L, Chang WS, Zubarev ER, Nordlander P, Link S. Influence of cross sectional geometry on surface plasmon polariton propagation in gold nanowires. ACS Nano. 2014;8:572-580.

[34] Chew WC, Jin JM, Michielssen E, Song J. Fast and efficient algorithms in computational electromagnetics. Boston: Artech House; 2001.

[35] Ergül Ö, Gürel L. The multilevel fast multipole algorithm (MLFMA) for solving largescale computational electromagnetics problems. Chichester, West Sussex, UK: WileyIEEE; 2014.

[36] Çekinmez A, Karaosmanoğlu B, Ergül Ö. Integral-equation formulations of plasmonic problems in the visible spectrum and beyond. In Reyhanoğlu M, editor. Dynamical systems - analytical and computational techniques. InTech; 2017. 
[37] Karaosmanoğlu B, Yımaz A, Ergül Ö. On the accuracy and efficiency of surface formulations in fast analysis of plasmonic structures via MLFMA. In Proceedings of the Progress in Electromagnetics Research Symposium (PIERS); Shanghai, 2016, pp. 2629-2633.

[38] Karaosmanoğlu B, Yılmaz A, Gür UM, Ergül Ö. Solutions of plasmonic structures using the multilevel fast multipole algorithm. Int J RF Microw Comput. Aided Eng. 2016;26:335-341.

[39] Önol C, Karaosmanoğlu B, Ergül Ö. Efficient and accurate electromagnetic optimizations based on approximate forms of the multilevel fast multipole algorithm. IEEE Antennas Wirel Propag. Lett. 2016;15:1113-1115. 
